\title{
Suppression of FUT1 attenuates cell proliferation in the HER2-overexpressing cancer cell line NCI-N87
}

\author{
SADAYUKI KAWAI, SHUNSUKE KATO, HIROO IMAI, YOSHINARI OKADA and CHIKASHI ISHIOKA \\ Department of Clinical Oncology, Institute of Development, Aging and Cancer, Tohoku University, \\ Aoba-ku, Sendai 980-8575, Japan
}

Received February 15, 2012; Accepted April 23, 2012

DOI: $10.3892 /$ or.2012.2120

\begin{abstract}
Lewis Y (LeY) antigen is an oligosaccharide that is highly expressed at the cell surface in various human cancers. Increased LeY expression activates epidermal growth factor receptor (EGFR) and human epidermal growth factor receptor 2 (HER2) and promotes cell proliferation in EGFRoverexpressing cells. However, the effect of downregulation of LeY expression on cell proliferation in HER2-overexpressing cells remains unknown. FUT1 encodes $\alpha 1,2$-fucosyltransferase, a key enzyme for LeY synthesis. We knocked down FUT1 by short interfering RNA (siRNA) in four HER2-overexpressing human cancer cell lines, including NCI-N87, MKN7, SKBr3 and BT474. We investigated whether downregulation of LeY and alteration in the glycosylation status of these cells affect cell proliferation and HER2 activation. Knocking down FUT1 expression markedly inhibited proliferation of NCI-N87, which highly expressed EGFR and was sensitive to EGFR deprivation. Furthermore, FUT1 siRNA downregulated the total amount of HER2 protein, phosphorylation of HER2 and EGFR, and phosphorylation of extracellular signal-regulated kinase (ERK) in this cell line. Moreover, the marked downregulation of phosphorylation of HER2 and ERK was observed following short-time EGF-stimulation. These effects were not observed in the other three cell lines. Our results suggest that knockdown of FUT1 downregulates HER2 signaling via EGFR downregulation. FUT1 may serve as a new molecular target for HER2-overexpressing human cancers with activated EGFR signaling.
\end{abstract}

\section{Introduction}

The cell membrane of mammalian cells comprises glycolipids, glycoproteins and proteoglycans; these carbohydrate structures

Correspondence to: Dr Chikashi Ishioka, Department of Clinical Oncology, Institute of Development, Aging and Cancer, Tohoku University, 4-1 Seiryo-machi, Aoba-ku, Sendai 980-8575, Japan E-mail: chikashi@idac.tohoku.ac.jp

Key words: $\alpha 1,2$-fucosyltransferase, Lewis Y antigen, HER2overexpression, epidermal growth factor receptor, proliferation, short interfering RNA undergo conformational changes during cellular differentiation and transformation (1). In particular, protein glycosylation accounts for the vast majority of post-translational processes that affect protein folding, stability, solubility and function (2). Glycosylation is catalyzed by glycosyltransferases. Among them, fucosyltransferases transfer an L-fucose sugar from a GDP-fucose donor substrate to an acceptor substrate (3). The fucosyltransferase gene family encodes enzymes that transfer fucose from $\alpha(1,2), \alpha(1,3 / 4)$ and $\alpha(1,6)$ linkages to various glycans. FUT1 and FUT2 encode $\alpha(1,2)$-fucosyltransferases, which transfer a terminal fucose residue from an $\alpha(1,2)$-linkage to an existing galactose Type 1 or 2 precursor substance and form the $\mathrm{H} 1$ or $\mathrm{H} 2$ antigen as precursors of soluble $\mathrm{ABH}$ antigens, respectively (4). FUT1 is ubiquitously expressed in the human body and preferentially expressed in erythroid tissues and vascular endothelial cells. FUT2 is mainly expressed in the epithelial cells of the digestive and respiratory tracts (4). FUT3-FUT7 and FUT9 encode $\alpha(1,3)$-fucosyltransferases, and their gene products transfer a fucose residue from an $\alpha(1,3)$-linkage to galactose in $\mathrm{H} 1$ and $\mathrm{H} 2$ antigens to produce various Lewis antigens $(5,6)$.

Antigens of the ABH and Lewis histo-blood group family can be found on the cell surface of various normal cells, mainly epithelial cells. However, the expression of various carbohydrate epitopes of this family is altered in carcinomas (7). For example, Lewis Y antigen (LeY), a Lewis antigen, is expressed in various cancer cells, including breast, ovarian and colorectal cancer; its expression is often associated with poor prognosis (8-12). In addition, forced FUT1 and FUT2 expression in human ovarian carcinoma-derived RMG-I cells increases activity of $\alpha(1,2)$-fucosyltransferase and LeY antigen and promotes cell proliferation and resistance against anticancer drugs, such as 5-FU and carboplatin $(13,14)$.

The molecular mechanisms through which overexpression of LeY antigen induces a malignant phenotype remain to be elucidated. However, increased LeY expression induced by FUT1 and FUT2 overexpression activates the epidermal growth factor receptor (EGFR) signaling and induces increase in mRNA expression and protein levels of human epidermal growth factor receptor 2 (HER2), a member of EGFR family (15). Furthermore, an FUT1- and FUT2-overexpressing cell line proliferates more aggressively than the parent cell line (15), suggesting that activation of EGFR and HER2 induces a malignant phenotype in human cancer cells. 
HER2 is a transmembrane glycoprotein that is fucosylated by fucosyltransferase. It is involved in transmitting signals that stimulate cell division (16). HER2-overexpression is caused by amplification of the HER2 gene and it is observed in various cancers $(17,18)$, including breast and gastric cancer. Clinically, it is a molecular target of trastuzumab, a monoclonal antibody against HER2 (18,19). However, whether alteration of glycosylation affects cell proliferation in HER2-overexpressing cancer cells remains to be investigated. In this study, we examined the effect of FUT1 suppression on the HER2 pathway and cell proliferation.

\section{Materials and methods}

Cell lines and cell culture. In this study, we used four HER2overexpressing human cancer cell lines, NCI-N87 and MKN7 (derived from gastric cancer) and SKBr3 and BT474 (derived from breast cancer), and two cell lines, A431 and VMRC-LCD (derived from lung cancers), that do not overexpress HER2. NCI-N87, SKBr3, BT474 and VMRC-LCD were obtained from the American Type Culture Collection (Manassas, VA, USA). MKN7 and A431 were provided by the Cell Resource Center for Biomedical Research (Institute of Development, Aging and Cancer, Tohoku University, Sendai, Japan). All cell lines were maintained in RPMI-1640 medium (Sigma, St. Louis, MO, USA) supplemented with $10 \%$ heat-inactivated FBS (Gibco, Grand Island, NY, USA) and incubated at $37^{\circ} \mathrm{C}$ in a $5 \% \mathrm{CO}_{2}$ humidified atmosphere.

Preparation of siRNA and transfection. The following three pairs of siRNA oligomers were designed according to the sequence of human FUT1 (GenBank accession number: NM_000148):FUT1-1 siRNA,5'-AAAGGAUCUCUCAAGUC CGCGTT-3' and 5'-CGCGGACUUGAGAGAUCCUUUTT-3'; FUT1-2 siRNA, 5'-GCUACACCGUGGAAAGACUTT-3' and 5'-AGUCUUUCCACGGUGUAGCTT-3'; FUT1-3 siRNA, 5'-UCGAUGUUUUCUUUACACCAC-3' and 5'-GGUGUAA AGAAAACAUCGACA-3'.

FUT1-1 and FUT1-2 siRNAs were designed based on a previous report (20) and the resource of Open Biosystems (http://www.openbiosystems.com), respectively. FUT1-3 siRNA was designed using a web-based online software system (siDirect version 2.0, http://sidirect2.rnai.jp). These siRNAs were chemically synthesized by Hokkaido System Science, Co., Ltd. (Hokkaido, Japan).

Signal Silence EGF Receptor siRNA 1 and 2 (Santa Cruz Biotechnology, Inc., CA, USA) were used as EGFR-siRNA1 and 2, respectively. Negative control siRNA (Silencer Negative Control no. 1 siRNA) was obtained from Ambion, Inc. (Austin, TX, USA). Cells were seeded in a 6- or 96-well plate. After $24 \mathrm{~h}$, the cells were transfected with siRNA (100 nM final concentration) using Dharmafect 2 reagent (Dharmacon, Lafayette, CO, USA).

Measurement of $m R N A$ expression by real-time PCR. Realtime polymerase chain reaction (PCR) was used to measure the mRNA expression of FUT1 in the cells. Cells were plated at $1.5 \times 10^{4}$ in a 96 -well plate and transfected with siRNA (100 nM final concentration) as described above. Twentyfour hours post-transfection, the total-RNA from the cells

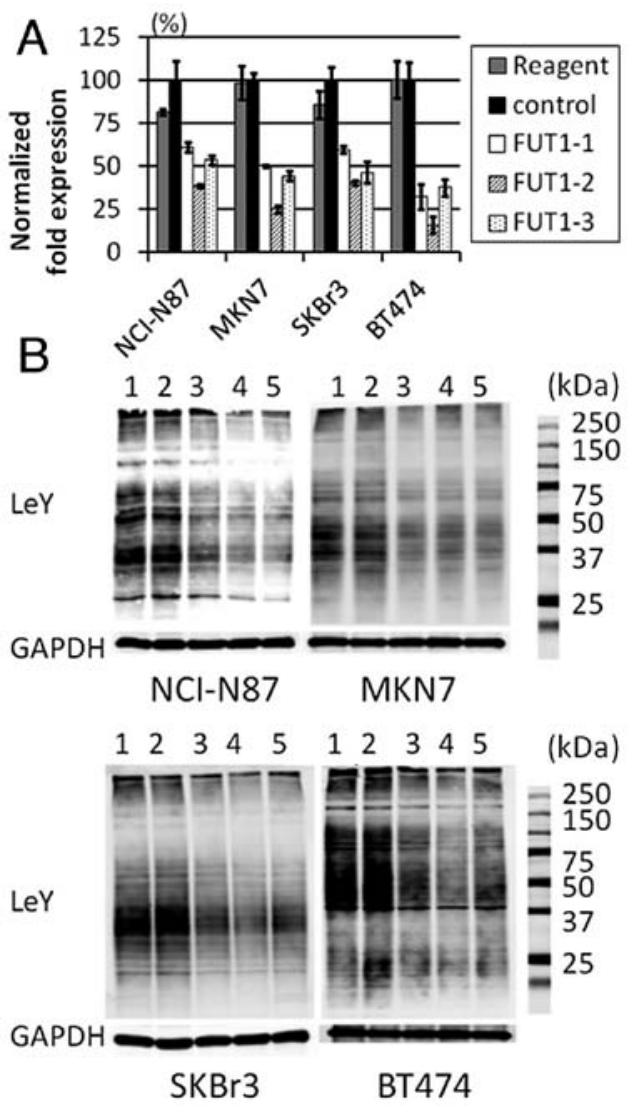

Figure 1. (A) Suppression of FUT1 mRNA expression after transfection of siRNAs was analyzed by real-time PCR. The level of FUT1 mRNA expression was determined and plotted as fold change relative to the control. Data were normalized to the amount of glyceraldehyde 3-phosphate dehydrogenase (GAPDH) mRNA. (B) Alteration of LeY expression after transfection of siRNAs in each whole cell lysate. GAPDH is shown as a loading control. Lane 1, reagent; lane 2, control; lane 3, FUT1-1; lane 4, FUT1-2; and lane 5, FUT1-3. A molecular weight standard is shown at the right side.

was extracted using the RealTime ready cell lysis kit (Roche Diagnostics GmbH, Mannheim, Germany) and reverse transcribed using the Transcriptor First Strand cDNA synthesis kit (Roche Diagnostics GmbH) with oligo(dt) primer. Real-time PCR was performed using SsoFast ${ }^{\mathrm{TM}}$ EvaGreen Supermix (Bio-Rad, Richmond, CA, USA) and gene-specific primers in a thermal cycler CFX96 real-time PCR detection system (Bio-Rad). The primers used for amplification were FUT1 F, 5'-AACGCCTCCTCTTCCTGTC-3' and R, 5'-TGGGG TAGACAGTCCAGGTG-3'; glyceraldehyde 3-phosphate dehydrogenase (GAPDH) F, 5'-GAAGGTGAAGGTCG GAGTC-3' and R, 5'-GAAGATGGTGATGGGATTTC-3' (GenBank accession no. NM_002046). The designs of both primers have been previously described (21). Quantified data were normalized to GAPDH. The PCR program included 45 cycles of $95^{\circ} \mathrm{C}$ for $1 \mathrm{sec}$ and $60^{\circ} \mathrm{C}$ for $5 \mathrm{sec}$. After the PCR reaction was completed, a melting curve analysis was performed. Each primer pair produced a single and sharp peak, thereby indicating that the primers amplified only one specific PCR product. No primer dimers were observed. All samples were amplified in triplicate.

Western blotting. Cells were plated at $2.0 \times 10^{5}$ in a 6 -well plate and transfected with siRNA (100 $\mathrm{nM}$ final concentration) 
FUT1-1
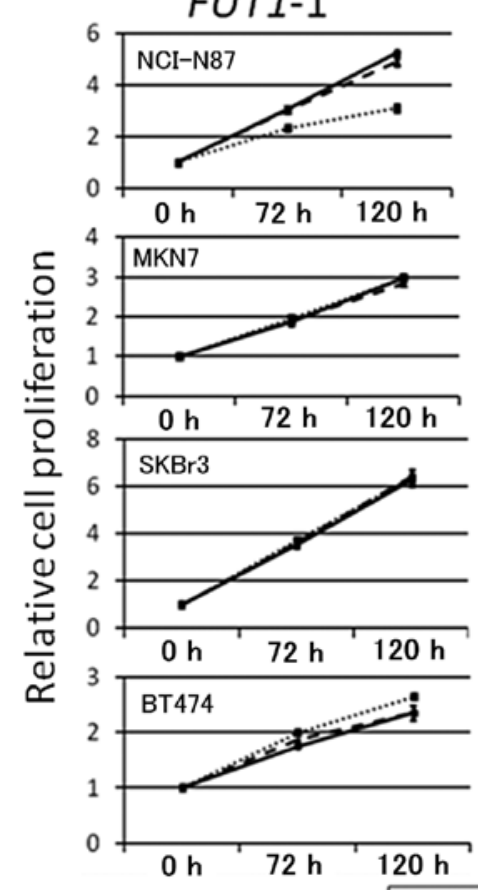

FUT1-2
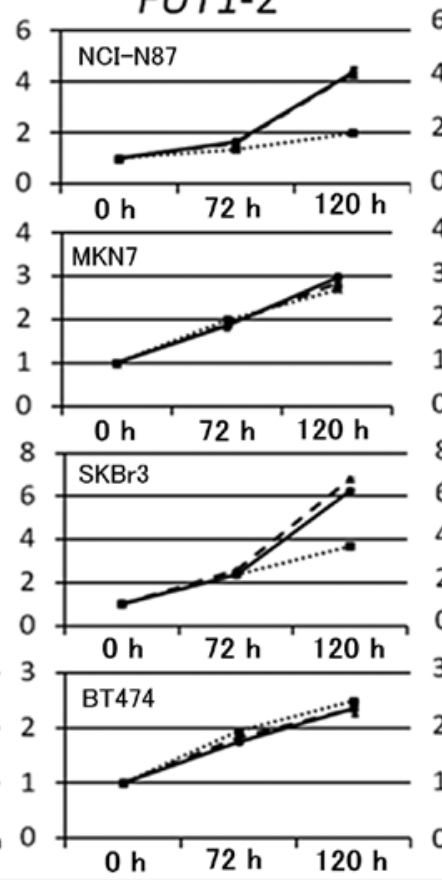

Reagent $-\Delta-\operatorname{control} \cdots \cdot . \cdot$ FUT1 siRNA
FUT1-3
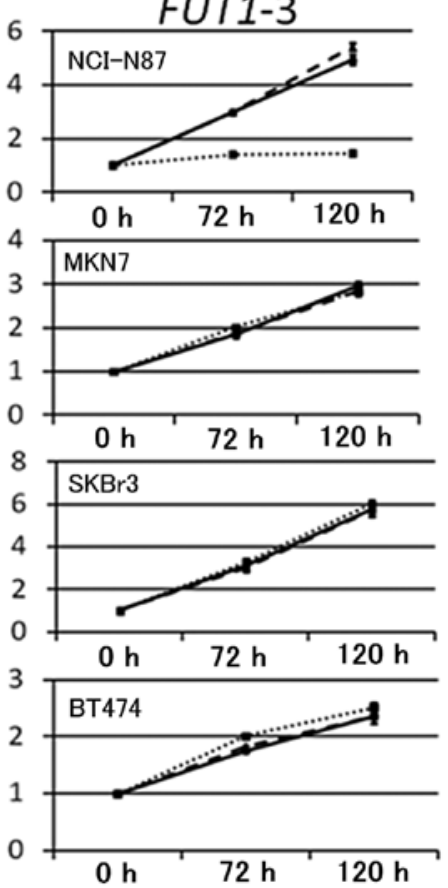

Figure 2. The growth curves after transfection with FUT1 siRNA. Cells were assayed for growth 0,72 and $120 \mathrm{~h}$ after transfection. The absorbance at $450 \mathrm{~nm}$ at $0 \mathrm{~h}$ was normalized to one. Absorbance at subsequent time points was plotted relative to the initial value.

as described above. After $72 \mathrm{~h}$, the cells were washed with cold PBS and harvested with lysis buffer [50 $\mathrm{mM}$ Tris- $\mathrm{HCl}$ (pH 8.0), $150 \mathrm{nM} \mathrm{NaCl}, 5 \mathrm{nM}$ EDTA, 1\% NP-40, protease inhibitor cocktail (Roche Diagnostics $\mathrm{GmbH}$ ) and phosphatase inhibitor (Roche Diagnostics $\mathrm{GmbH}$ )]. In the short-time EGF stimulation experiment, $72 \mathrm{~h}$ post-transfection, the cells were starved in serum-free medium for $12 \mathrm{~h}$ and stimulated with EGF (10 ng/ml) for $10 \mathrm{~min}$. The cells were washed immediately and harvested as described above.

Cell lysates were separated by $12.5 \%$ SDS-PAGE and blotted onto a PVDF membrane. The membranes were blocked with the Odyssey blocking buffer (Li-Cor Biosciences, Lincoln, NE, USA) and then probed with polyclonal antiHER 2 antibody (Dako, Carpinteria, CA, USA), monoclonal anti-phosphorylated HER2 antibody (Tyr1248; Santa Cruz Biotechnology, Inc.), monoclonal anti-EGFR antibody (Santa Cruz Biotechnology, Inc.), monoclonal anti-phosphorylated EGFR antibody (Tyr1068), monoclonal anti-GAPDH antibody (Santa Cruz Biotechnology, Inc.), anti-ERK1/2 antibody (Cell Signaling Technology, Inc.), anti-phosphorylated ERK1/2 antibody (Thr202/Tyr204; Cell Signaling Technology, Inc.) and anti-LeY antibody (Abcam, Cambridge, UK), followed by incubation with a goat anti-rabbit or a goat anti-mouse Alexa Fluor $680 \mathrm{IgG}$ secondary antibody (Invitrogen, Carlsbad, CA, USA). Protein bands were detected and quantified using the Odyssey system (Li-Cor Biosciences).

Cell proliferation assay. Cells were plated at $3.0 \times 10^{3}$ in a $96-$ well plate and transfected with siRNA (100 $\mathrm{nM}$ final concentration) as described above. At 0,72 and $120 \mathrm{~h}$ the cells were harvested and cell viability was determined using the Cell Counting kit-8 (Dojin Laboratories, Kumamoto, Japan), which measures mitochondrial succinate dehydrogenase activity. Briefly, $10 \mu 1$ of 2-(2-methoxy-4-nitrophenyl)-3-(4-nitrophenyl)-5-(2,4disulfophenyl)-2H-tetrazolium monosodium salt (WST-8) solution was added to each well. After a 2-h incubation at $37^{\circ} \mathrm{C}$, the resulting water-soluble formazan dye was assayed by a microplate autoreader SpectraMax (Molecular Devices, Sunnyvale, CA, USA) at a wavelength of $450 \mathrm{~nm}$ with a reference of $630 \mathrm{~nm}$. All experiments were performed in triplicate.

Cell cycle analysis using fuorescence-activated cell sorter (FACS). Cells were plated at $2.0 \times 10^{5}$ in a 6 -well plate and transfected with siRNA (100 $\mathrm{nM}$ final concentration) as described above. After a 72-h incubation, cells were treated with trypsin and fixed with $70 \%$ ethanol in PBS overnight. The cells were then washed once with PBS, incubated in the presence of RNase A $(0.25 \mathrm{mg} / \mathrm{ml})$ for $30 \mathrm{~min}$ at $37^{\circ} \mathrm{C}$, collected by centrifugation at $200 \mathrm{x}$ g for $5 \mathrm{~min}$ and stained with propidium iodide $(50 \mu \mathrm{l} / \mathrm{ml})$. The cells were filtered through a $50-\mu \mathrm{m}$ pore size nylon mesh and analyzed for cell cycle using a FACS system (Beckman Coulter, Miami, FL, USA).

Statistical analysis. All experiments were performed independently and in triplicate. Data are expressed as means \pm standard error. Statistical data were analyzed using Student's t-test. Significance was set at $\mathrm{P}<0.05$.

\section{Results}

FUT1 knockdown by siRNA decreases LeY antigen expression. To detect the effects of FUT1 siRNAs, we performed real-time PCR analysis (Fig. 1A) and western blotting of LeY antigen (Fig. 1B). FUT1 mRNA and LeY antigen expression were 


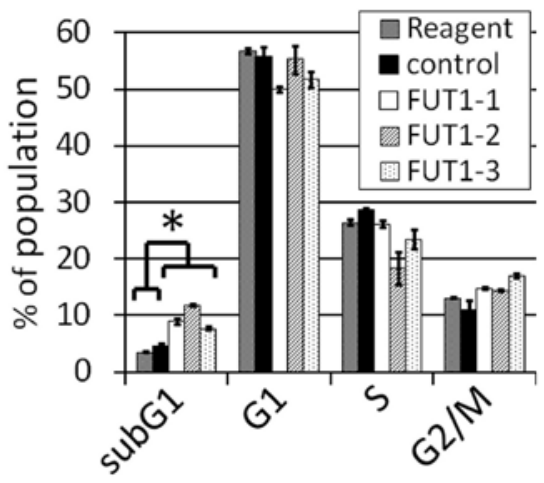

Figure 3. Analysis of the proportion of cells in each cell cycle phase after transfection with each FUT1 siRNA in NCI-N87 cells. Values shown are average $\pm \mathrm{SE}(\mathrm{n}=3)$. Values of the sub-G1, G1, S and G2/M fractions are portions of the total population. " $\mathrm{P}<0.01$

reduced in all cells transfected with FUTI siRNAs, but not in those transfected with control siRNA. Compared with the cells transfected with control siRNA, the level of LeY expression in the cells transfected with FUT1-1, FUT1-2 or FUT1-3 siRNAs was FUT1-1 67.9, FUT1-2 44.5 and FUT1-3 46.9\% in NCI-N87 cells; FUT1-1 50.5, FUT1-2 58.6 and FUT1-3 51.7\% in MKN7 cells; FUT1-1 49.2, FUT1-2 33.3 and FUT1-3 62.3\% in SKBr3 cells; and FUT1-1 54.1, FUT1-2 41.1 and FUT1-3 58.8\% in BT474 cells. These results indicated that FUT1 siRNAs efficiently reduced the levels of FUT1 mRNA and LeY antigen expression.

FUT1 knockdown inhibits cell proliferation in NCI-N87 cells, but not in other cell lines. To examine the effect of siRNAmediated FUT1 knockdown on cell growth, cell proliferation assays were performed for the four HER2-overexpressing cell lines. Data are shown in Fig. 2. FUT1 siRNAs inhibited NCI-N87 cell proliferation $120 \mathrm{~h}$ post-transfection, whereas they did not inhibit proliferation in MKN7 or BT474 cells. Although FUT1-2 suppressed proliferation in $\mathrm{SKBr} 3$ cells, FUT1-1 and FUT1-3 did not. Therefore this was considered to be an off-target siRNA effect.

FUT1 knockdown leads to apoptosis in NCI-N87 cells. To examine whether $F U T 1$ knockdown changes the proportion of cells in each cell cycle phase, FACS analysis was performed for NCI-N87 cells (Fig. 3). All siRNAs significantly increased the subG1 fraction $(\mathrm{P}<0.01)$. The $\mathrm{G} 2 / \mathrm{M}$ fraction also increased, but not significantly $(\mathrm{P}=0.09)$. These results indicate that the downregulation of $F U T 1$ mRNA and LeY antigen expression leads to apoptosis in NCI-N87 cells.

FUT1 knockdown downregulates the expression of HER2 and the phosphorylated HER2 (pHER2) and phosphorylated ERK1/2 (pERK) in NCI-N87 cells. To elucidate the mechanism of cell growth inhibition induced by FUT1 knockdown, HER2,

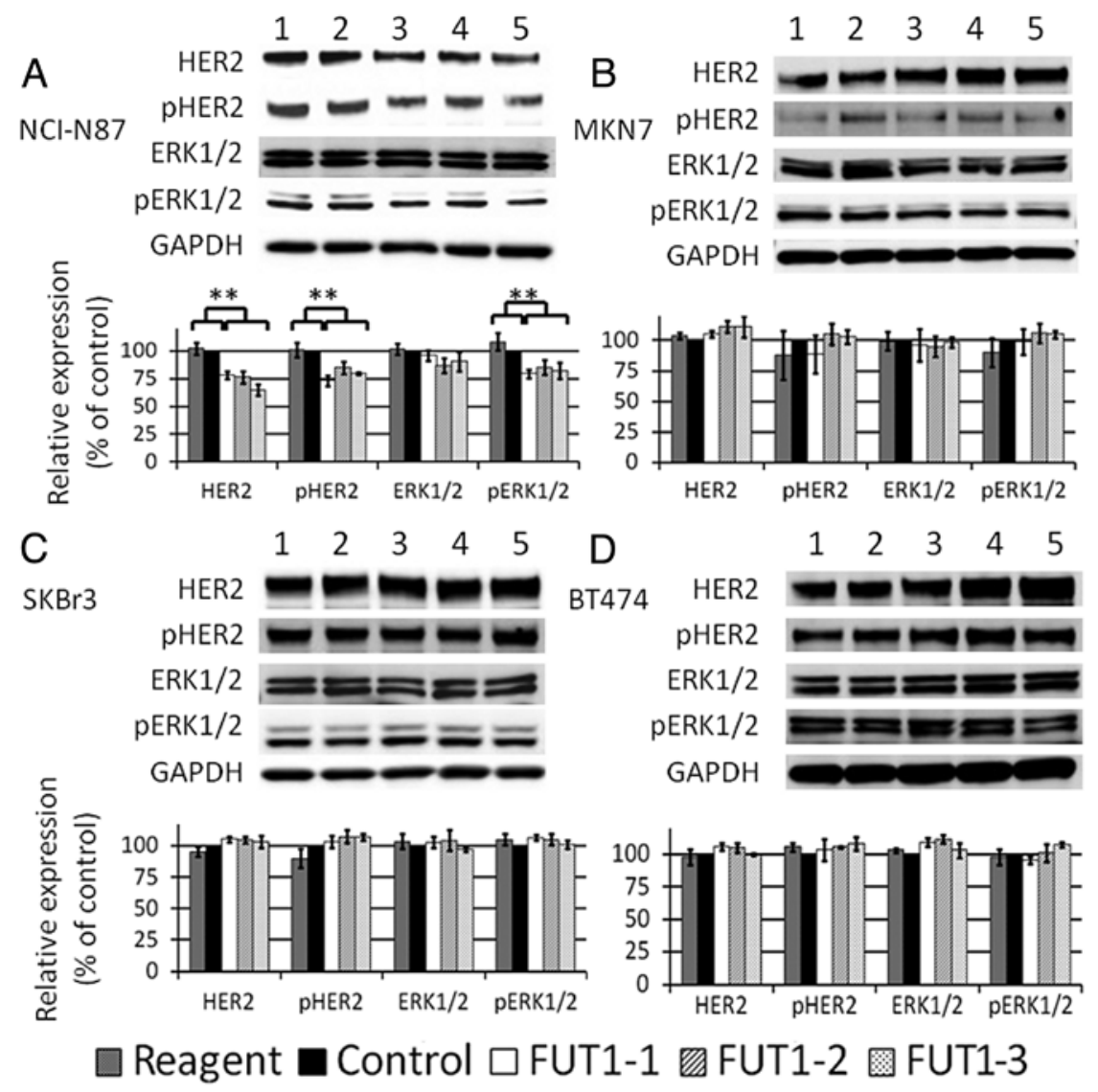

Figure 4. The effect of downregulating FUT1 on protein expression and tyrosine phosphorylation of HER2 and ERK1/2 in normal culture condition. The bar chart shows the relative expression ratio of each protein by western blotting. Each value is calculated as the ratio of signal intensity compared to that of control. Data were normalized to signal intensity of glyceraldehyde 3-phosphate dehydrogenase (GAPDH). Lane 1, reagent; lane 2, control; lane 3, FUT1-1; lane 4, FUT1-2; and lane 5, FUT1-3. (A) NCI-N87, (B) MKN7, (C) SKBr3 and (D) BT474 cells. ${ }^{* *} \mathrm{P}<0.05$. 


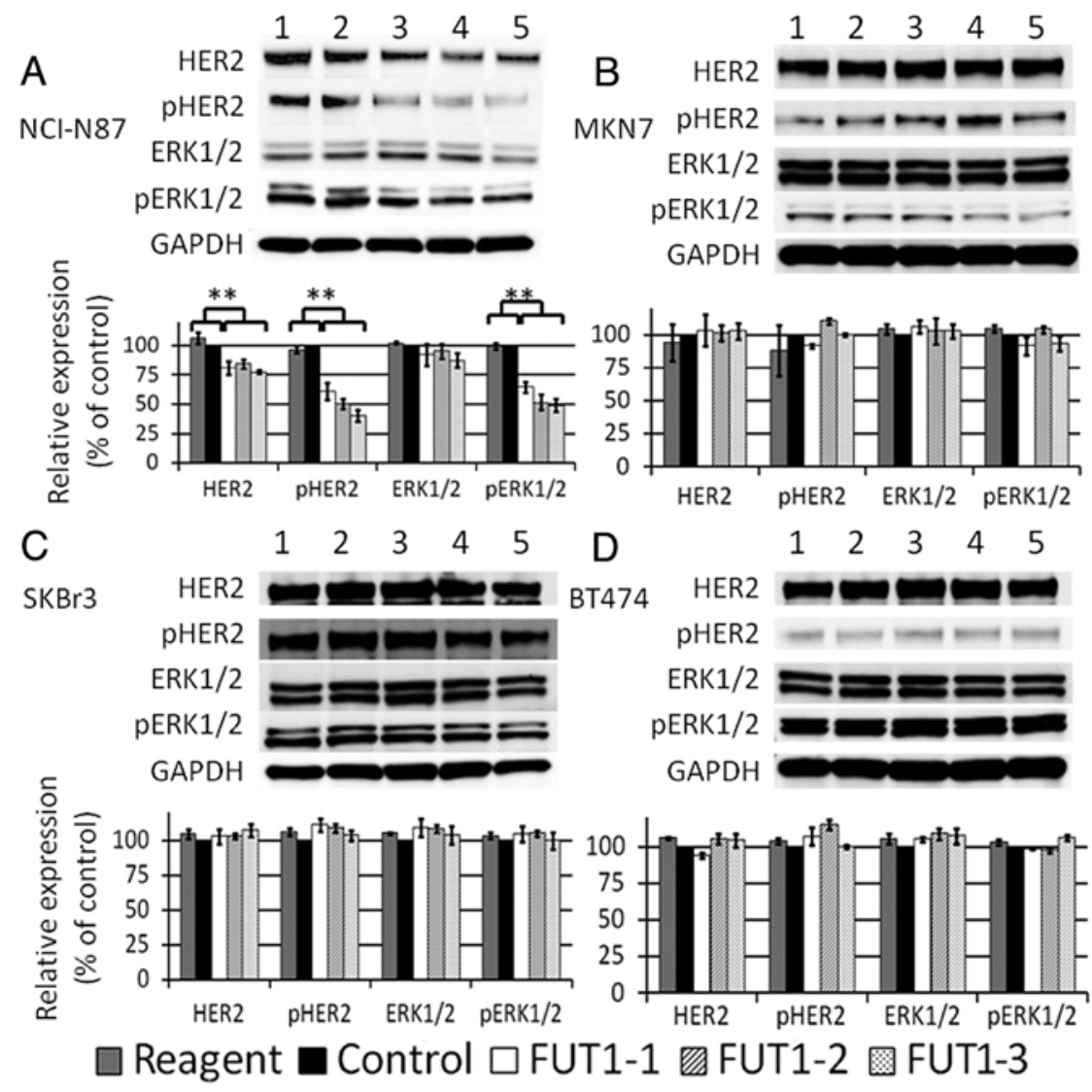

Figure 5. The effect of downregulating FUT1 on protein expression and tyrosine phosphorylation of HER2 and ERK1/2 under EGF-stimulated condition. The bar chart shows the relative expression ratio of each protein found by western blotting. Each value is calculated as the ratio of signal intensity compared to that of control. Data were normalized to signal intensity of glyceraldehyde 3-phosphate dehydrogenase (GAPDH). Lane 1, reagent; lane 2, control; lane 3, FUT1-1; lane 4, FUT1-2; and lane 5, FUT1-3. (A) NCI-N87, (B) MKN7, (C) SKBr3 and (D) BT474 cells. ** $\mathrm{P}<0.05$.

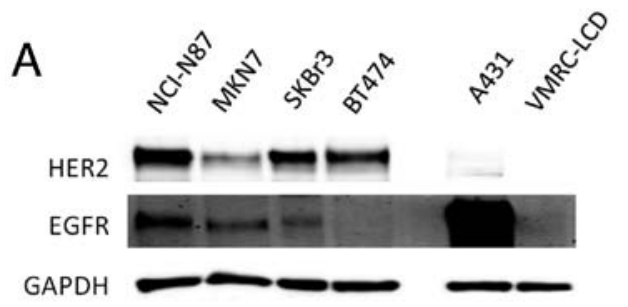

B

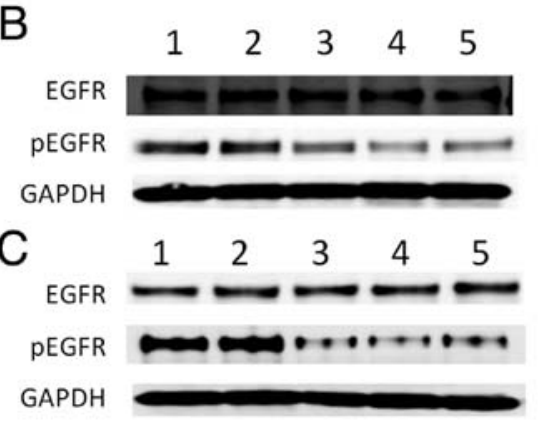

Figure 6. (A) HER 2 and EGFR expression in each cell line was analyzed by western blotting. A431 is shown as a positive control for EGFR-overexpression and VMRC-LCD is shown as a negative control. (B) The effect of downregulating FUT1 on protein expression and tyrosine phosphorylation of EGFR under normal cultural condition in NCI-N87 cells. Glyceraldehyde 3-phosphate dehydrogenase (GAPDH) is shown as the loading control. (C) The effect of downregulating $F U T 1$ on protein expression and tyrosine phosphorylation of EGFR under EGF-stimulated condition in NCI-N87 cells. Glyceraldehyde 3-phosphate dehydrogenase (GAPDH) is shown as the loading control. Lane 1, reagent; lane 2, control; lane 3, FUT1-1; lane 4, FUT1-2; and lane 5, FUT1-3.
pHER2, ERK1/2 and pERK were assessed by western blotting $72 \mathrm{~h}$ after transfection (we called it 'normal cultural conditon').

Representative western blotting data and bar charts by triplicate experiments are shown in Fig. 4. FUT1 knockdown significantly downregulated the total amount of HER2 and pHER2 in NCI-N87 cells (Fig. 4A). The amount of pERK also decreased, but the total amount of ERK remained unchanged. In contrast to NCI-N87, no significant changes were observed in MKN7, SKBr3 or BT474 cells (Fig. 4B-D).

FUT1 knockdown strongly downregulates pHER2 and pERK following short-time EGF stimulation in NCI-N87 cells. To examine whether short-time EGF-stimulation alters downregulation of HER 2 and ERK1/2 by FUT1 knockdown, we administered EGF for 10 min after starvation of the cells and assessed the amount of HER2, pHER2, ERK1/2 and pERK. The amount of pHER 2 and pERK was markedly reduced in NCI-N87 cells (Fig. 5A). This reduction was more apparent than that of normal culture condition (Fig. 4A). Alterations in HER 2 and ERK1/2 levels were similar to those observed in normal culture condition. In contrast, no significant changes were observed following EGF stimulation in MKN7, SKBr3 or BT474 cells (Fig. 5B-D).

FUT1 knockdown downregulates EGFR signaling in NCI-N87. To examine whether FUT1 suppression affects EGFR signaling, first, western blotting for EGFR expression 

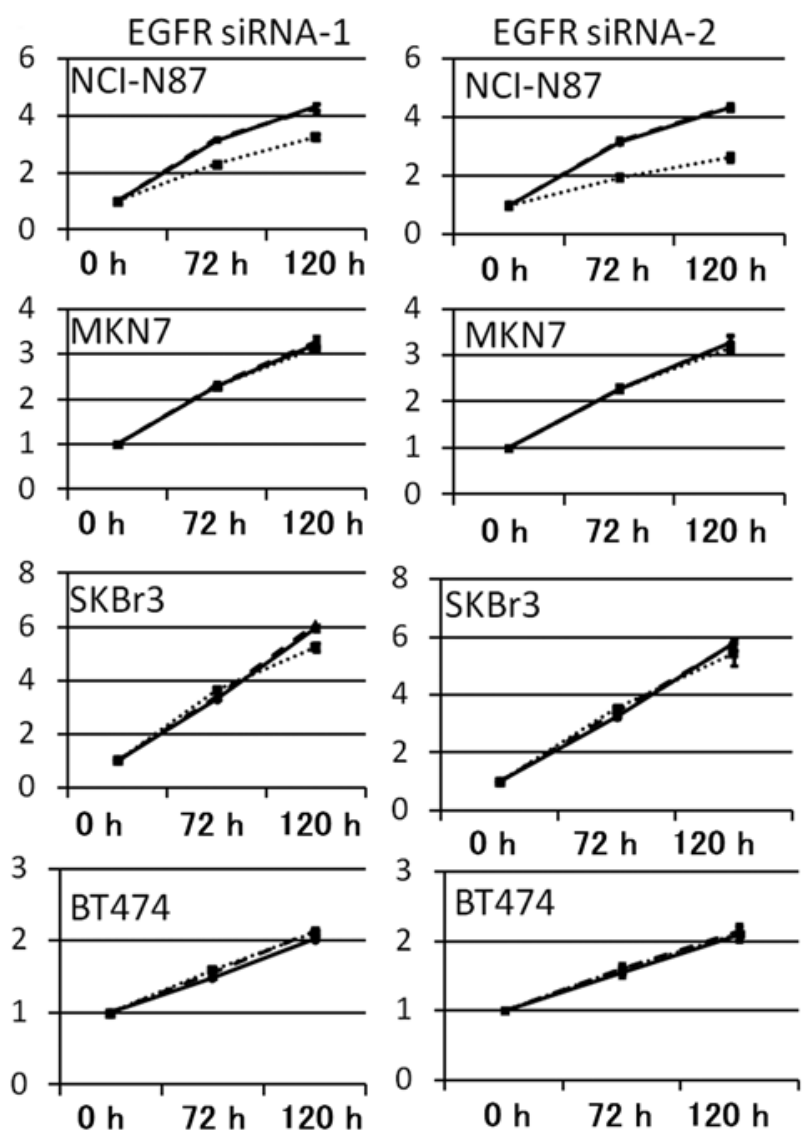

$\rightarrow$ Reagent $\leadsto-$ control .... EGFR siRNA

Figure 7. The growth curves after transfection with EGFR siRNA-1 and EGFR siRNA-2. Cells were assayed for growth 0,72 and $120 \mathrm{~h}$ after transfection. The absorbance at $450 \mathrm{~nm}$ at $0 \mathrm{~h}$ was normalized to one. Absorbance at subsequent time points was plotted relative to the initial value.

was performed (Fig. 6A). EGFR expression in each cell line was lower than that in the EGFR-overexpressing A431 cell line, whereas EGFR expression in NCI-N87 was higher than that in other HER2-overexpressing cell lines. Then, we investigated EGFR signaling of NCI-N87 after FUT1 suppression. The result showed that phosphorylation of EGFR was downregulated in both normal cultural and EGF-stimulating conditions. The total amount of EGFR did not change in either of the conditions (Fig. 6B and C).

Suppression of EGFR signaling downregulates HER2 signaling and proliferation of NCI-N87 cells. To confirm the proliferation dependence of EGFR signaling the cell lines were transfected with EGFR siRNA. EGFR siRNA-1 and EGFR siRNA-2 suppressed the proliferation of NCI-N87 cells by 23.6 and $44.7 \%$, respectively. However the proliferation of other cell lines were not changed by EGFR knockdown (Fig. 7). Next, to investigate whether EGFR suppression affects HER2 signaling in NCI-N87 cells, western blotting was performed. EGFR suppression downregulated HER2, pHER2 and pERK as well as FUT1 suppression in NCI-N87 cells (Fig. 8). The results indicated that the proliferation of NCI-N87 cells was also dependent on EGFR signaling and EGFR suppression resulted in downregulation of HER 2 signaling in this cell line.

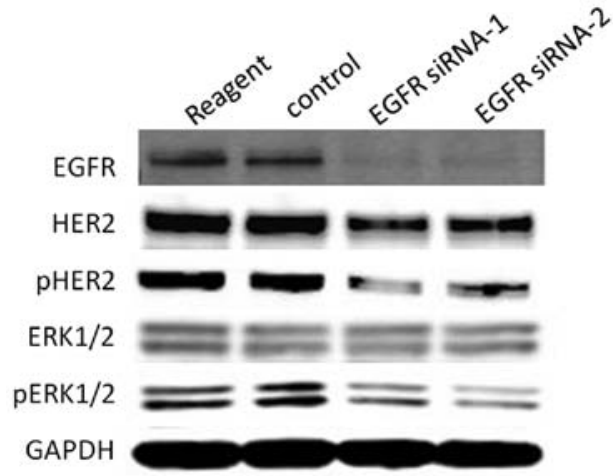

Figure 8. The effect of EGFR knockdown on protein expression and tyrosine phosphorylation of HER2 and ERK1/2 in NCI-N87 cells. Glyceraldehyde 3 -phosphate dehydrogenase (GAPDH) is shown as the loading control.

\section{Discussion}

LeY antigen belongs to the histo-blood group antigens and a1,2-fucosyltransferase is the key enzyme, which also FUT1 and FUT2 encode. Previous studies suggested that forced expression of $\alpha 1,2$-fucosyltransferase in RMG-I human ovarian cancer cell line caused overexpression of LeY antigen and promoted cell proliferation via activation of EGFR and HER2 (15). Furthermore, Palumberi et al (20) indicated that suppression of $\alpha 1,2$-fucosyltransferase inhibited the cell proliferation of the EGFR-overexpressing cell line A431. In the present study, we attempted to suppress FUT1 gene by its specific siRNA and observe whether FUT1 knockdown affected the cell proliferation of HER2-overexpressing cell lines.

Our results indicated that FUT1 siRNA downregulated FUT1 mRNA and altered fucosylation; it was shown by inhibition of LeY antigen expression, in four HER2-overexpressing cell lines. However, the effects on cell proliferation varied. In NCI-N87 cells, FUT1 suppression decreased the total amount of HER2, pHER2 and pERK, and inhibited cell proliferation. However, FUT1 suppression in MKN7, SKBr3 and BT474 did not alter HER2, pHER2 or pERK levels and did not affect cell proliferation.

In a previous study, HER 2 inhibition led to suppression of cell proliferation in HER2-overexpressing cell lines (22-24). This observation is similar to our results and indicates that HER2 plays an important role in cell proliferation in HER2overexpressing cells.

In addition, our study suggested that EGFR signaling was involved in FUT1-mediated inhibition of HER2 signaling in NCI-N87 cells. The experiment of EGFR siRNA transfection indicated that the proliferation of NCI-N87 cells was depentdent not only on HER2 signaling but also on EGFR signaling and EGFR suppression led to HER2 signaling inhibition. Previous studies indicated that cetuximab, a monoclonal antibody against EGFR, inhibits cell proliferation in NCI-N87 cells (25), but not in SKBr3 or BT474 cells (24). These results suggest that EGFR potently contributes to the proliferation of NCI-N87 cells.

We speculate that FUT1 suppression leads to HER2 inhibition and cell proliferation via EGFR signaling inhibition through one or both mechanisms described below. 
First, downregulation of EGFR by FUT1 suppression may attenuate HER2 transcription. Liu et al (15) reported that FUT1-overexpression upregulated EGFR signaling and increased mRNA expression and protein levels of HER2. In our study, FUT1 knockdown decreased the total amount of HER 2 in NCI-N87 cells. Therefore, FUT1 knockdown may have decreased HER2 levels by downregulating EGFR signaling. Since the level of attenuation of the total amount of HER2 was similar to that of pHER2, a reduction of the total amount of HER2 may cause downregulation of pHER2 and pERK in normal culture condition.

Second, FUT1 suppression may attenuate EGFR and HER2 heterodimer formation. HER2 forms homodimers or heterodimers with other EGFR family proteins, undergoes autophosphorylation at specific tyrosine residues of its intracellular domain and mediates signal transduction (17). In addition, EGFR forms homodimers or heterodimers with other EGFR family proteins following ligand stimulation (25).

Following starvation and short-time EGF stimulation, phosphorylation of HER 2 and ERK1/2 was markedly reduced in FUT1-suppressed NCI-N87 cells. Zhang et al (26) reported that the suppression of FUT1 and FUT4 reduced LeY antigen, decreased binding of EGF to EGFR and resulted in inhibition of cell proliferation. In addition, some reports have shown that fucosylation on EGFR alters the binding affinity of EGF to EGFR and affects EGFR dimerization $(2,27,28)$.

Hence, we propose that FUT1 suppression caused an alteration of fucosylation and attenuated EGF-mediated EGFR and HER2 heterodimerization.

Besides, our results indicated that apoptosis occurred in FUT1-mediated growth inhibition in NCI-N87 cells. G2/M fraction also tended to increase but not significantly. Previous studies revealed that HER2 inhibition by trastuzumab caused apoptosis in some HER2-overexpressing cell lines, e.g. SKBr3 or Calu-3 (29). However, it did not cause apoptosis in SKOV-3 which had HER2-overexpression (30). Hence, it is possible that HER2 suppression causes various effects on cell proliferation among each cell line.

Lapatinib is a dual tyrosine kinase inhibitor for EGFR and HER2 and is used to treat trastuzumab-resistant HER2 positive cancers. Redundant signaling from other EGFR family members is one of the molecular mechanisms of drug resistance to trastuzumab (31). Inhibition of EGFR and HER2 signaling is one strategy for treating trastuzumab-resistant HER2 positive cancers.

The role of fucosylation in cell proliferation is not completely understood. However, our results demonstrate that FUT1 knockdown results in the inhibition of cell proliferation and reduction of HER2, pHER2 and pERK in NCI-N87 cells. The reduction of pHER 2 and pERK seems to depend on the reduction of EGFR signaling caused by inhibition of fucosylation. Further studies are necessary to identify a biomarker to predict which HER2-positive cancer cells are sensitive to FUT1 inhibition. The development of a fucosyltransferase inhibitor may constitute a novel drug for trastuzumab-resistant HER2 positive cancers.

\section{Acknowledgements}

The authors thank Satoko Aoki for her technical assistance.

\section{References}

1. Roseman S: Reflections on glycobiology. J Biol Chem 276: 41527-41542, 2001.

2. Liu YC, Yen HY, Chen CY, et al: Sialylation and fucosylation of epidermal growth factor receptor suppress its dimerization and activation in lung cancer cells. Proc Natl Acad Sci USA 108: 11332-11337, 2011.

3. Javaud C, Dupuy F, Maftah A, et al: The fucosyltransferase gene family: an amazing summary of the underlying mechanisms of gene evolution. Genetica 118: 157-170, 2003.

4. Matzhold EM, Helmberg W, Wagner T, et al: Identification of 14 new alleles at the fucosyltransferase 1,2, and 3 loci in Styrian blood donors, Austria. Transfusion 49: 2097-2108, 2009.

5. Dettke M, Pálfi G and Loibner H: Activation-dependent expression of the blood group-related Lewis Y antigen on peripheral blood granulocytes. J Leukoc Biol 68: 511-514, 2000.

6. Hokke CH, Neeleman AP, Koeleman CA and van den Eijinden DH: Identification of an $\alpha 3$-fucosyltransferase and a novel $\alpha 2$-fucosyltransferase activity in cercariae of the schistosome Trichobilharzia ocellata: biosynthesis of the Fuca1->2Fuc $\alpha 1-->3[\mathrm{Gal}(\mathrm{NAc})$ (1-->4] GlcNAc sequence. Glycobiology 8: 393-406, 1998.

7. Nakagoe T, Fukushima K, Itoyanagi N, et al: Expression of $\mathrm{ABH} /$ Lewis-related antigens as prognostic factors in patients with breast cancer. J Cancer Res Clin Oncol 128: 257-264, 2002.

8. Tsuboi K, Asao T, Ide M, et al: $\alpha 1,2$-fucosylation is a superior predictor of postoperative prognosis for colorectal cancer compared with blood group A, B, or sialyl Lewis X antigen generated within colorectal tumor tissue. Ann Surg Oncol 14: 1880-1889, 2007.

9. Madjd Z, Parsons T, Watson NF, Spendlove I, Ellis I and Durrant LG: High expression of Lewis $y / b$ antigens is associated with decreased survival in lymph node negative breast carcinomas. Breast Cancer Res 7: R780-R787, 2005.

10. Arai $Y$ and Nishida M: Differential diagnosis between normal endometrium and endometrial hyperplasia with immunostaining cytology using anti-LeY monoclonal antibody. Int J Gynecol Cancer 13: 42-46, 2003.

11. Kim YS, Yuan M, Itzkowitz SH, et al: Expression of LeY and extended LeY blood group-related antigens in human malignant, premalignant, and non-malignant colonic tissues. Cancer Res 46: 5985-5992, 1986.

12. Kitamura K, Stockert E, Garin-Chesa P, et al: Specificity analysis of blood group Lewis-y (Le(y)) antibodies generated against synthetic and natural Le(y) determinants. Proc Natl Acad Sci USA 91: 12957-12961, 1994.

13. Iwamori M, Tanaka K, Kubushiro K, et al: Alterations in the glycolipid composition and cellular properties of ovarian carcinoma-derived RMG-1 cells on transfection of the $\alpha 1$, 2-fucosyltransferase gene. Cancer Sci 96: 26-30, 2005.

14. Zhao Y, Lin B, Hao YY, et al: The effects of Lewis(y) antigen content on drug resistance to carboplatin in ovarian cancer line RMG-I. Prog Biochem Biophys 35: 1175-1182, 2008.

15. Liu JJ, Lin B, Hao YY, et al: Lewis(y) antigen stimulates the growth of ovarian cancer cells via regulation of the epidermal growth factor receptor pathway. Oncol Rep 23: 833-841, 2010.

16. Brennan PJ, Kumogai T, Berezov A, et al: HER2/Neu: mechanisms of dimerization/oligomerization. Oncogene 19: 6093-6101, 2000.

17. Slamon DJ, Godolphin W and Jones LA: Studies of the HER-2/ neu proto-oncogene in human breast and ovarian cancer. Science 244: 707-712, 1989.

18. Gravalos C and Jimeno A: HER2 in Gastric Cancer: A New Prognostic Factor and a Novel Therapeutic Target. Ann Oncol 19: 1523-1529, 2008.

19. Owens MA, Horten BC and Da Silva MM: HER2 amplification ratios by fluorescence in situ hybridization and correlation with immunohistochemistry in a cohort of 6556 breast cancer tissues. Clin Breast Cancer 5: 63-69, 2004.

20. Palumberi D, Aldi S, Ermini L, et al: RNA-mediated gene silencing of FUT1 and FUT2 influences expression and activities of bovine and human fucosylated nucleolin and inhibits cell adhesion and proliferation. J Cell Biochem 111: 229-238, 2010.

21. Chang WW, Lee $\mathrm{CH}$, Lee $\mathrm{P}$, et al: Expression of Globo $\mathrm{H}$ and SSEA3 in breast cancer stem cells and the involvement of fucosyl transferases 1 and 2 in Globo H synthesis. Proc Natl Acad Sci USA 105: 11667-11672, 2008 
22. Mittendorf EA, Liu Y, Tucker SL, et al: A novel interaction between HER2/neu and cyclin E in breast cancer. Oncogene 29: 3896-3907, 2010.

23. Tanner M, Hollmén M, Junttila TT, et al: Amplification of HER-2 in gastric carcinoma: association with Topoisomerase IIa gene amplification, intestinal type, poor prognosis and sensitivity to trastuzumab. Ann Oncol 16: 273-278, 2005.

24. Brockhoff G, Heckel B, Schmidt-Bruecken E, et al: Differential impact of Cetuximab, Pertuzumab and Trastuzumab on BT474 and SK-BR-3 breast cancer cell proliferation. Cell Prolif 40: 488-507, 2007.

25. Patel D, Bassi R, Hooper A, et al: Anti-epidermal growth factor receptor monoclonal antibody cetuximab inhibits EGFR/HER-2 heterodimerization and activation. Cancer Sci 99: 1611-1617, 2008.

26. Zhang Z, Sun P, Liu J, et al: Suppression of FUT1/FUT4 expression by siRNA inhibits tumor growth. Biochim Biophys Acta 1783: 287-296, 2008.
27. Miyoshi E, Moriwaki K and Nakagawa T: Biological function of fucosylation in cancer biology. J Biochem 143: 725-729, 2008.

28. Wang $\mathrm{X}, \mathrm{Gu} \mathrm{J}$, Ihara $\mathrm{H}$, et al: Core fucosylation regulates epidermal growth factor receptor-mediated intracellular signaling. J Biol Chem 281: 2572-2577, 2006.

29. Dogan I, Cumaoglu A, Aricioglu A and Ekmekci A: Inhibition of ErbB2 by herceptin reduces viability and survival, induces apoptosis and oxidative stress in Calu-3 cell line. Mol Cell Biochem 347: 41-51, 2011.

30. Bijman MN, van Berkel MP, Kok M, Janmaat ML and Boven E: Inhibition of functional HER family members increases the sensitivity to docetaxel in human ovarian cancer cell lines. Anticancer Drugs 20: 450-460, 2009.

31. Kruser TJ and Wheeler DL: Mechanisms of resistance to HER family targeting antibodies. Exp Cell Res 316: 1083-1100, 2010. 